\title{
Bird strike on aircraft radome: Dynamic characterisation of quartz fibre composite sandwich for accurate, predictive impact simulations
}

\author{
Sebastian Heimbs ${ }^{1, *}$, Tim Wagner ${ }^{2}$, Heinz Meister ${ }^{3}$, Clemens Brand $^{3}$, and Mircea Calomfirescu ${ }^{3}$ \\ ${ }^{1}$ Airbus Operations, 21129 Hamburg, Germany \\ ${ }^{2}$ Airbus Central R\&T, 81663 Munich, Germany \\ ${ }^{3}$ Airbus Defence and Space, 85077 Manching, Germany
}

\begin{abstract}
This study assesses the bird strike resistance of the satellite communication (SatCom) radome of a medium altitude, long endurance (MALE) remotely piloted aircraft system (RPAS), which is designed as a lightweight sandwich structure with thin quartz fibre composite skins and a cellular honeycomb core. In order to perform accurate, predictive numerical bird strike simulations, the building block approach was applied, involving extensive experimental characterisation and model validation of the materials and structures from simple coupon level up to full-scale radome level. Coupon tests of the quartz fibre composite skin material under high-rate dynamic loading revealed significant strain rate effects, which needed to be taken into account in the simulation model in order to predict the structural response under high-velocity bird strike loading. In summary, this work presents a systematic and detailed approach for obtaining validated modelling methods for high-velocity impact analyses, which could be used efficiently for various design and parameter studies during the development of the SatCom radome.
\end{abstract}

\section{Introduction}

Aircraft radomes are electro-magnetically transparent, structural enclosures of radar antennas that can both be found on commercial as well as military aircraft (Fig. 1). Being forward-facing structures, bird strike is one of the major design-driving load cases for such radomes and bird strike resistance for specific impact masses and velocities needs to be shown before allowance for operational use [1]. This has typically been done experimentally in the past and is more and more replaced by sufficiently validated numerical impact simulations today [2-4].
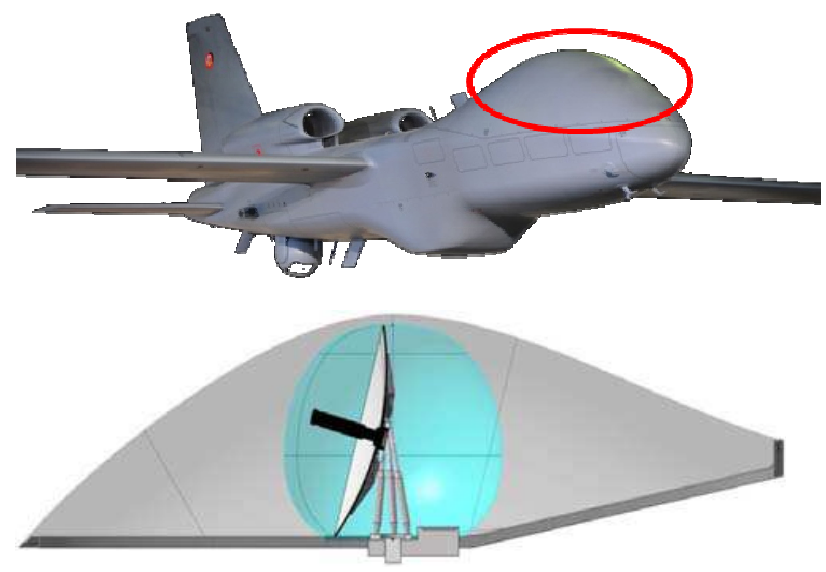

Fig. 1. SatCom radome of MALE RPAS.
Within this study the target was to develop bird strike simulation models of a SatCom radome made of composite sandwich structures in the commercial finite element software Abaqus/Explicit. An established method to achieve high accuracy in simulative predictions of structural behaviour of large, complex components is the so-called building block approach, which is based on a typical test pyramid (Fig. 2). Extensive tests for material characterisation on coupon level are performed to obtain knowledge on material behaviour and parameters for constitutive modelling. These models are then applied and validated with test data from the next higher level, typically element or component test. This approach is followed to the top of the pyramid, which is the full-scale structure. If the level of accuracy in the test-simulation correlations on the lower levels was high, then the accuracy of the numerical prediction of the full-scale structure can also be expected to be high.

This building block approach was applied for the bird strike simulation study of the SatCom radome and is presented in detail as follows. The focus was on dynamic tests on coupon level to assess any strain rate effects of the quartz fibre composite skin and Nomex ${ }^{\circledR}$ honeycomb materials and on impact tests on simplified sandwich structures. The models have been validated step by step to enable accurate predictions of the global response and design studies of the SatCom radome under bird strike loading.

*Corresponding author: sebastian.heimbs@airbus.com 


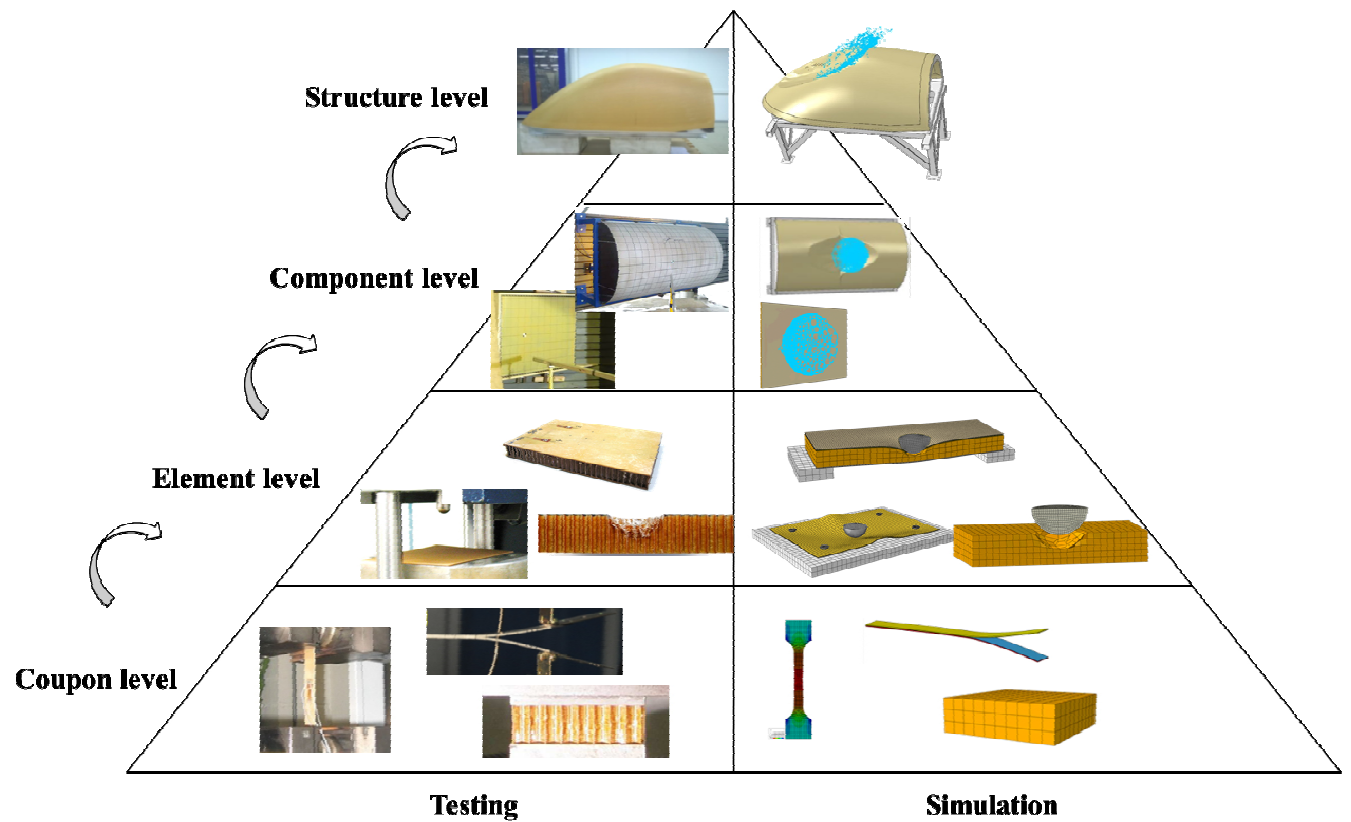

Fig. 2. Building block approach from coupon to structure level for accurate simulation models for bird strike analysis of radome.

\section{Material characterisation and modelling on coupon level}

The radome is made of a sandwich structure with a honeycomb core and quartz fibre composite skins. This material selection allows for a very light and electromagnetically transparent structure. The connection to the aircraft fuselage is designed as a monolithic composite interface (Fig. 3). Extensive coupon tests have been performed to characterise the material properties of skin and core, separately.

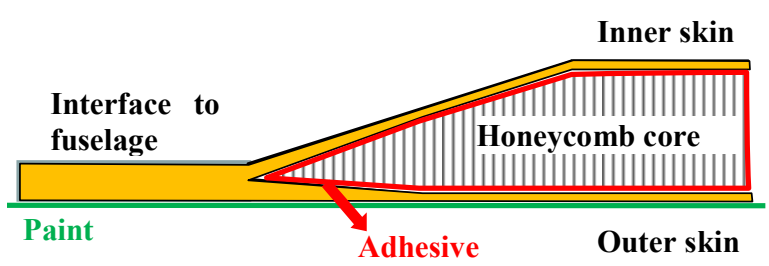

Fig. 3. Sandwich structure of SatCom radome.

\subsection{Quasi-static and dynamic characterisation of quartz fibre composite skins}

The composite sandwich skins are made of quartz silica fibres in 8-harness satin woven fabric configuration with 8552 epoxy resin. The following coupon tests were performed in order to obtain essential material properties and stress-strain curves: tensile tests in warp and weft direction (DIN EN ISO 527-4 T3), shear tests (AITM 1-0002), compression tests (AITM 10008 A1), double-cantilever beam (DCB) tests (AITM 10005) and end-notched flexure (ENF) tests (AITM 10006), see Fig. 4. The latter tests for interlaminar fracture energies are necessary for accurate delamination modelling.

The tensile and shear tests have been performed under quasi-static (strain rate $2 \cdot 10^{-4} \mathrm{~s}^{-1}$ ) and high-rate dynamic loading conditions (strain rates $20 \mathrm{~s}^{-1}$ and $80 \mathrm{~s}^{-1}$ ) on a servo-hydraulic testing machine. Significant strain rate effects were observed that lead to strength increases under high loading rates both for the fibre- and matrixdominated load cases (tension and shear, respectively), see Fig. 5. It is essential to include these strain rate effects in the simulation model in order not to underpredict material strength and failure.

Material modelling of the composite skins in Abaqus was based on 8-node continuum shell elements for each individual ply with the continuum damage mechanicsbased material model Abq_Ply_Fabric and cohesive contact interfaces in-between to capture delamination failure. This modelling approach allows for the representation of all relevant intra- and interlaminar failure modes of the skins. Finally, the coupon tests were simulated in order to verify the correct material properties.
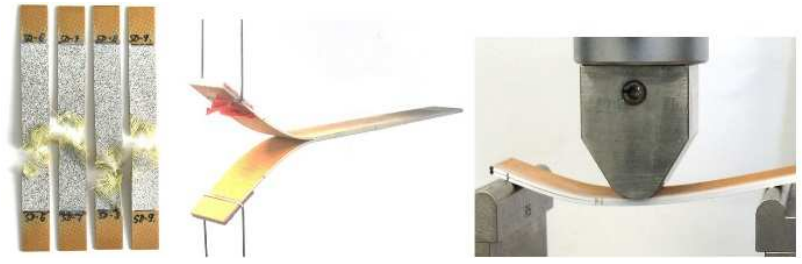

Fig. 4. Coupon testing of composite skin material.

\subsection{Quasi-static and dynamic characterisation of honeycomb core}

The sandwich core structure consists of a cellular Nomex $^{\circledR}$ honeycomb. The core was tested under compression, tension and shear loads in all directions (Fig. 6). These nonlinear, experimental stress-strain curves are the basis for the homogenised, orthotropic material modelling in Abaqus using Abq_Honeycomb for 8-node solid elements. In contrast to the composite skins, strain rate effects in the Nomex ${ }^{\circledR}$ honeycomb core 

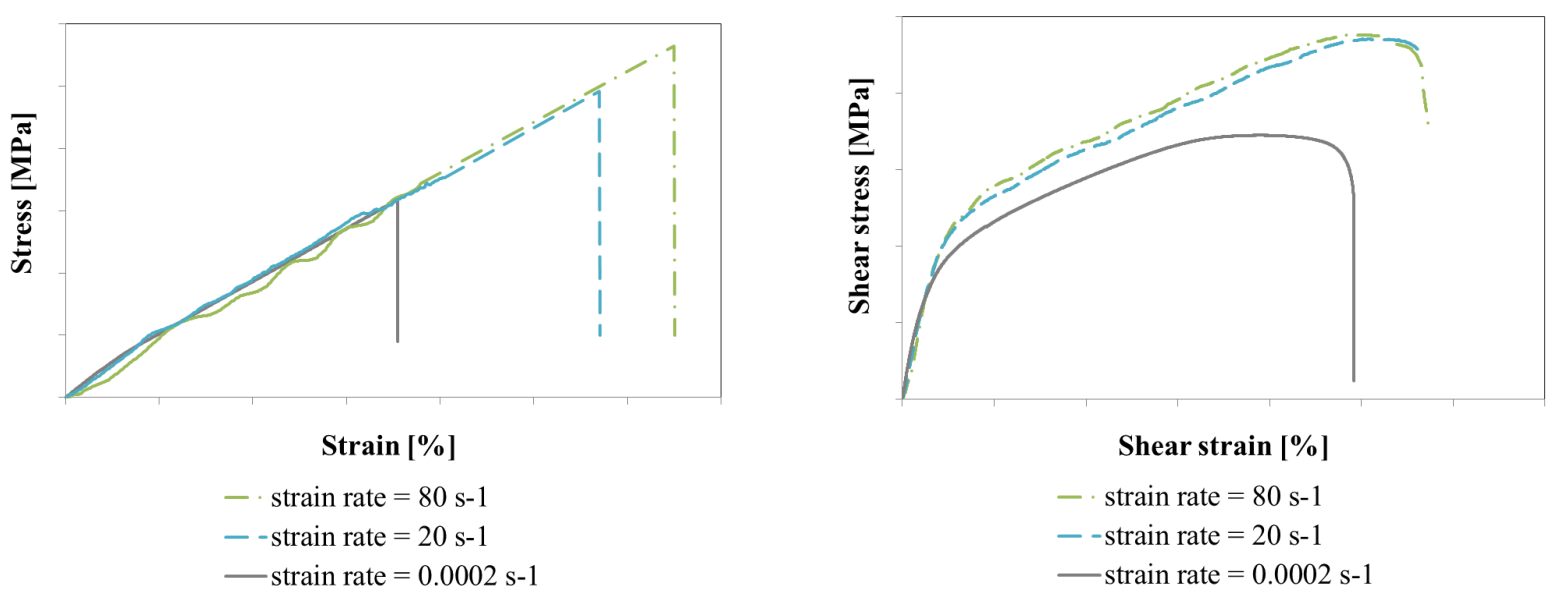

Fig. 5. Influence of strain rate on tensile (left) and shear (right) behaviour of quartz fibre composite.

plays a minor role with no significant increase of crushing strength (Fig. 7) [5]. Hence, they have been neglected in the constitutive model. Again, all these coupon tests have been simulated for verification and validation of the models and to assess any mesh size effects.
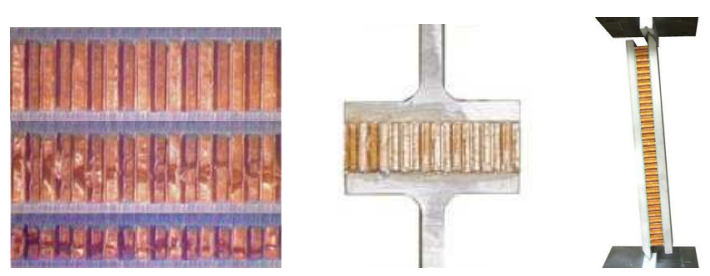

Fig. 6. Coupon testing of honeycomb core material.

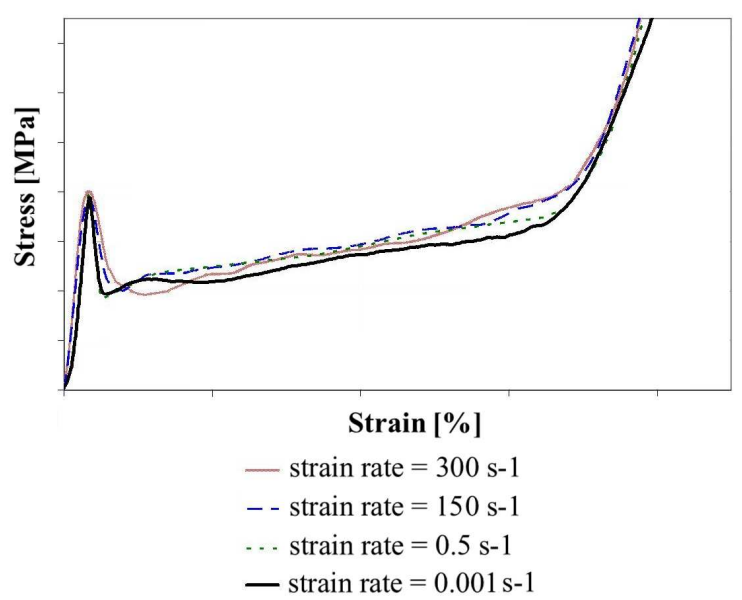

Fig. 7. Influence of strain rate on compression behaviour of honeycomb core.

\section{Low-velocity impact on sandwich materials}

The next step in the model development and experimental validation was to investigate the behaviour of skin, core and sandwich specimens under drop-weight impact. Performing such tests separately for the individual constituents of the sandwich structure allows for separate model validations under simple impact loading conditions of limited complexity.

\subsection{Low-velocity impact on composite skins}

The drop-weight impact tests of the skins were performed on thin quartz fibre composite plates with the actual sandwich skin thickness. Hence, the relatively low impact energies were varied in the range from $3 \mathrm{~J}$ to $20 \mathrm{~J}$ to generate various relevant states of damage incl. matrix cracking, delaminations and fibre rupture. The intra- and interlaminar damage models derived from the coupon tests could accurately represent the extent and mode of skin damage without any parameter calibrations (Fig. 8). Also the quantitative correlation of force-displacement plots between test and simulation was very promising.
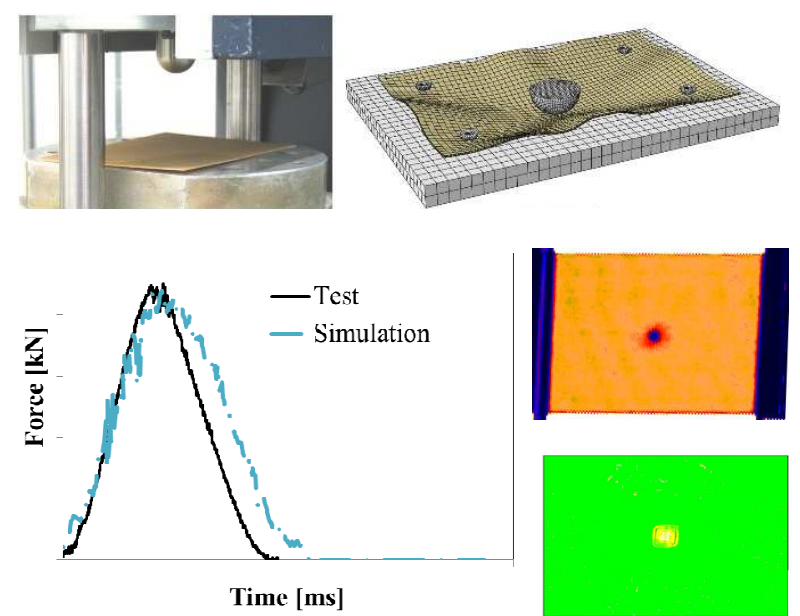

Fig. 8. Low-velocity impact on composite skins: testing and simulation, with force-time plot and C-scan vs. delamination contact visualisation.

\subsection{Low-velocity impact on honeycomb core}

The indentation behaviour of the honeycomb core was also tested and simulated separately (Fig. 9). In a mesh convergence study, the most suitable modelling method could be identified to accurately represent the core indentation and force-displacement response. 


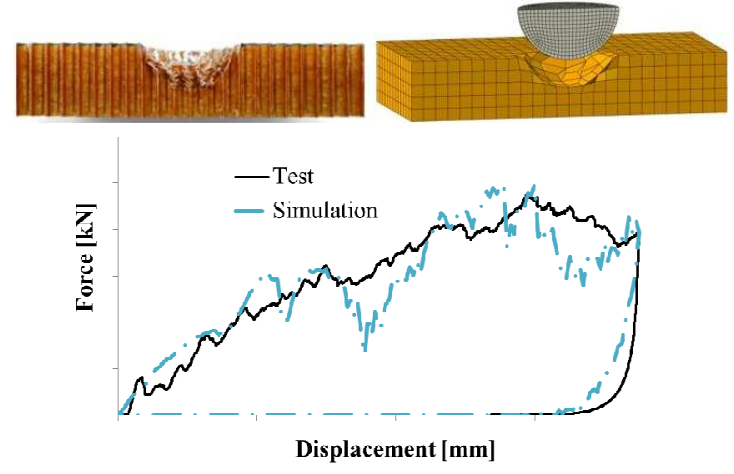

Fig. 9. Low-velocity impact on honeycomb core: testing and simulation, with force-displacement plot.

\subsection{Low-velocity impact on sandwich panel}

Finally, the validated skin and core models were combined in a low-velocity impact simulation of sandwich panels, validated with test data (Fig. 10). Most attention was paid to accurately predict the damage mode (i.e. core crushing and core shear failure) and size as well as the force-displacement behaviour. The good correlation gave confidence to use these modelling methods also for high-velocity bird impact simulations.
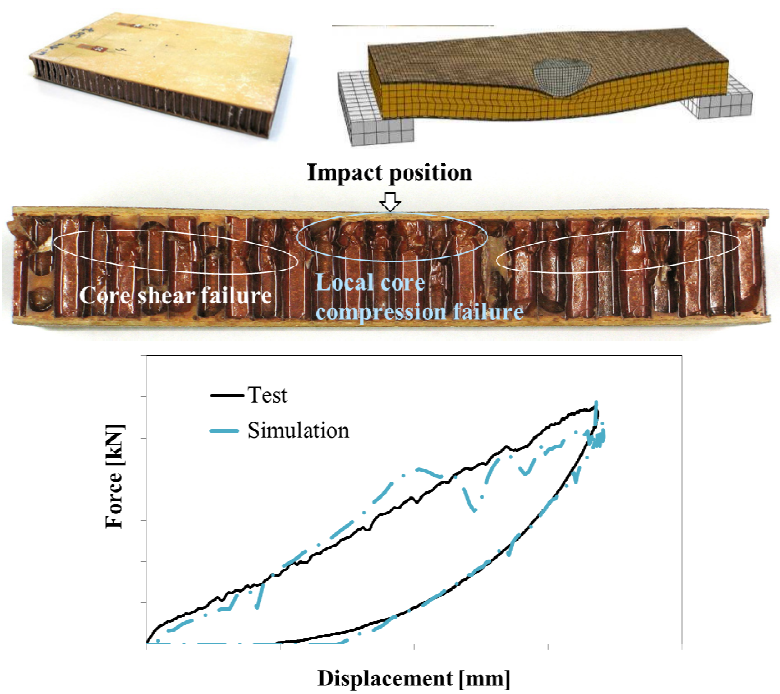

Fig. 10. Low-velocity impact on sandwich structure: testing and simulation, with cross-section for damage assessment and force-displacement plot.

\section{High-velocity bird impact on sandwich panels}

Before analysing the complex SatCom radome sandwich structure under bird strike loading, meaningful simplified intermediate steps were introduced to assess the bird strike loading on structures with less complexity and to provide further sources for model validations in the framework of the simulation method development. The complexity was increased step by step with bird impact tests on flat instrumented rigid plates, on flat sandwich panels and on single-curved sandwich panels.

\subsection{Bird strike on instrumented rigid plate}

To ensure that not only the model of the sandwich structure but also the model of the bird projectile is accurately validated, bird strike tests on instrumented rigid walls (Fig. 11) are a common way to compare pressure-time histories and flowing behaviour of the soft body projectile between test and simulation [6]. The bird in this study was idealised as a cylindrical body with two hemispherical ends, which is the common simplified representation of the torso for bird strike simulations [1]. The mesh-free smoothed particle hydrodynamics (SPH) method was applied, which avoids any mesh distortion issues of the highly deforming body. The bird behaves like a fluid at the velocities of interest, making an equation of state (EOS) for water with porosity an appropriate material modelling method. Using dedicated available bird impact tests for validation, the projectile behaviour could be simulated with high accuracy.
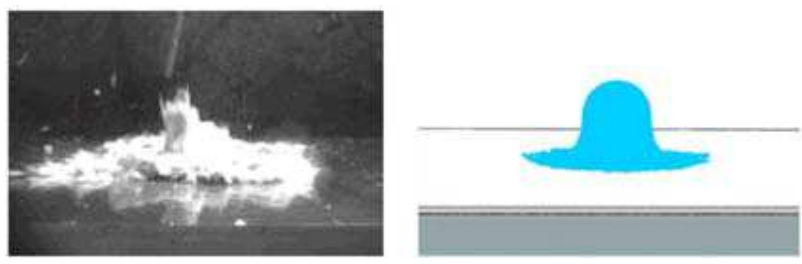

Fig. 11. High-velocity bird impact on instrumented rigid plate: testing and simulation.

\subsection{Bird strike on flat sandwich panel}

Bird strike tests on $1.2 \mathrm{~m}$ long flat sandwich panels with the identical material specification and lay-up as in the SatCom radome were performed experimentally and numerically (Fig. 12). Using the validated modelling methods for the sandwich structure and the bird projectile, the simulative prediction of damage mode (mainly core shear damage), extent of damage and plate deflection were in excellent correlation to the test data. An important lesson learned was that if quasi-static strength properties of the skin material were used, neglecting the strain rate effect, then the simulation predicted penetration and structural failure. Only with the inclusion of strain rate effects and the associated strength increase, accurate predictions were possible. The simulation results revealed that the strain rates of the composite skins in the impact zone were typically in the range of 20-200 s $\mathrm{s}^{-1}$.

\subsection{Bird strike on single-curved sandwich panel}

The next test campaign used a $2 \mathrm{~m}$ long single-curved sandwich panel as a pre-stage to the complex doublecurved SatCom radome (Fig. 13). During the simulations of this large structure it became obvious that the boundary conditions and the mounting frame of the large specimen also needed to be modelled in detail to capture its elastic response and influence on the global behaviour of the sandwich structure. Neglecting this by using simplified boundary conditions led to an underestimation of panel deflection. The numerically predicted damage 
was compared with ultrasonic C-scans, micro-CT scans and cut out cross-sections and it was in very good agreement. The dominating structural damage mode was core shear failure. Finally, all the simulation methods were regarded as sufficiently validated to be used for design studies of the SatCom radome structure.
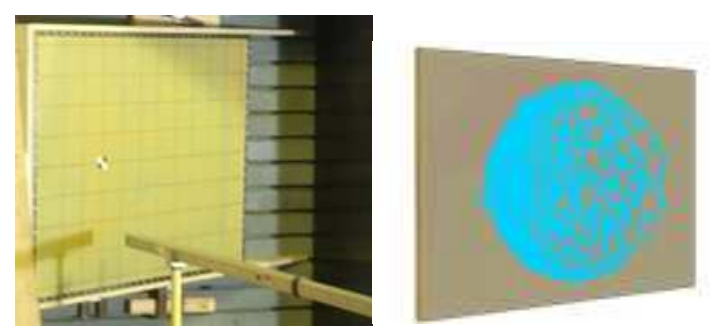

Fig. 12. High-velocity bird impact on flat sandwich panel: testing and simulation.
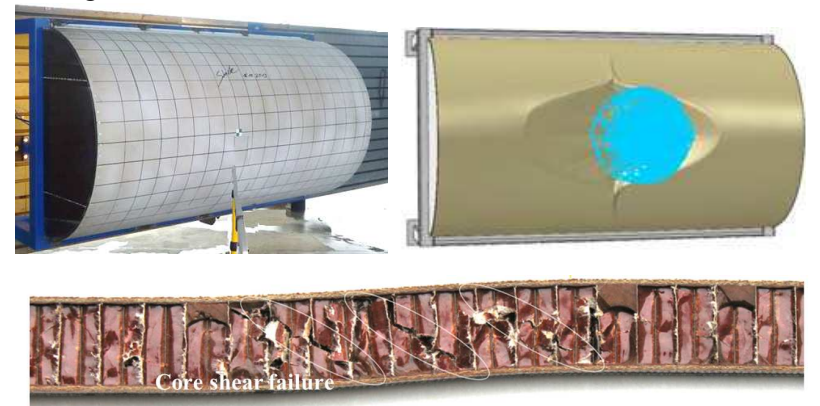

Fig. 13. High-velocity bird impact on curved sandwich panel: testing and simulation, with cross-section for damage analysis.

\section{High-velocity bird impact on aircraft radome}

The model development of the final full-scale SatCom radome structure using Abaqus was based on all the aforementioned, validated modelling methods for the sandwich materials and bird projectile. The model also incorporates the inner antenna for collision analysis as well as the metallic support structure used in the real bird strike test. While all previous tests have been performed in the context of simulation method development, verification and validation, the purpose of the final, physical bird strike test is different. The simulations are supposed to be used for efficient design and parameter studies of the radome to ensure bird impact resistance and to reduce any risk for the real test ("right first time"). The final bird strike test is intended to confirm the accuracy of the numerical predictions and to physically prove the impact resistance of the structural part. If this can be achieved, then these validated methods can also be used for further radome developments without the need for further physical tests, because the simulation model's robustness is sufficiently demonstrated.

The big advantage of accurate, robust simulation models is the possibility to efficiently perform parameter studies, which would not be feasible to be performed experimentally. A simulation parameter or geometrical design can quickly be changed and the impact simulation runs only a few hours, while the manufacturing and testing of prototypes would require significantly more budget and time. In this context, the influence of the following parameters was assessed in various simulation runs:

- influence of impact velocity,

- influence of impact angle (pitch and yaw angle),

- influence of (horizontal and vertical) impact position,

- influence of support structure stiffness,

- influence of sandwich material thickness,

- influence of manufacturing-induced splices and fibre angle variations by draping,

- influence of antenna in case of contact with radome.

With this approach the most critical load case in terms of bird impact position and angle could be identified, which leads to a maximum of structural damage (see Fig. 14). This was the basis for the final structural design of the radome and also for the specification of the physical test. Although a significant deformation of the sandwich structure occurs, which leads to extensive core shear failure, the composite skins do not break or detach and the numerical simulation predicts no loss of structural integrity of the radome.

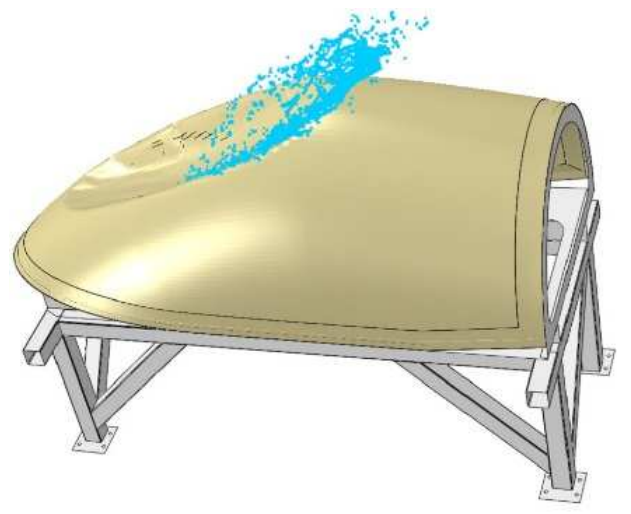

Fig. 14. High-velocity bird impact simulation of full-scale radome.

Finally, a $3 \mathrm{~m}$ long full-scale radome prototype (Fig. 15) was manufactured for a bird strike test to verify the bird impact resistance and the predictive quality of the numerical simulations, which was achieved successfully with good accuracy.

\section{Conclusion}

In summary, this work presents a systematic and detailed approach for obtaining validated modelling methods for high-velocity impact analyses, which could be used efficiently for various design and parameter studies during the development of the SatCom radome. The building block approach proved to be a meaningful method to develop and validate predictive modelling methods supported by tests with increasing complexity.

No later parameter calibrations have been necessary to achieve accurate results. The quartz fibre composite skin material of the radome sandwich structure showed 
significant strain rate effects with increasing strength under high-rate dynamic loading, which needed to be covered in the model for accurate predictions of the structural response under bird impact.

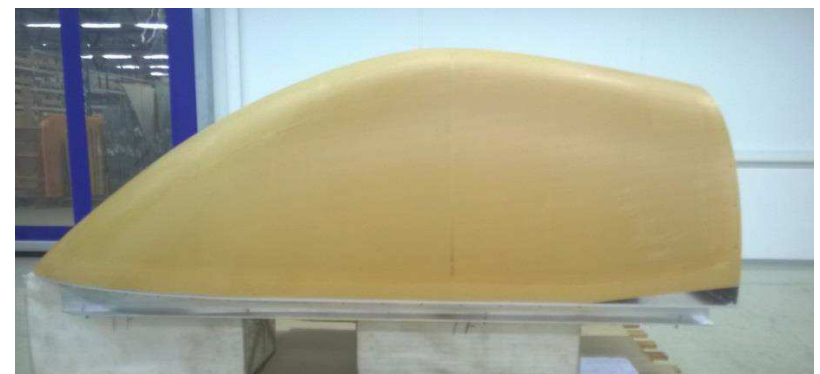

Fig. 15. Full-scale radome prototype for high-velocity bird impact test.

This building block approach is the appropriate method to generate accurate, predictive and validated numerical models for the transition from classical impact engineering solely based on expensive physical tests to efficient virtual testing (supported by few selected tests).
The authors would like to thank the various partners in the project consortium "FFS6 - Advanced aerostructures" that contributed to the development of the SatCom radome. The external testing partners involved in this study shall also be acknowledged, which are the Institute of Composite Materials (IVW) at Technical University of Kaiserslautern, Institute of Aerospace Engineering at Technical University of Dresden, WIWeB in Erding and IABG in Lichtenau.

\section{References}

1. S. Heimbs, Comput Struct 89, 2093-2112 (2011)

2. L. Wu, Y.N. Guo, Y. Li, Explosion and Shock Waves 29, 642-647 (2009)

3. X.Q. Zhang, X. Liu, Appl Mech Mater 63-64, 515$518(2011)$

4. Y.B. He, X.H. Yao, X. Liu, L.F. He, Appl Mech Mater 151, 305-309 (2012)

5. S. Heimbs, S. Schmeer, P. Middendorf, M. Maier, Compos Sci Technol 67, 2827-2837 (2007)

6. J. Liu, Y. Li, X. Gao, Int J Impact Eng 70, 21-37 (2014) 